\title{
An examination of high and low torque handpieces
}

\author{
High and low torque handpieces: cutting dynamics, enamel cracking and tooth temperature by T. F. Watson, \\ D. Flanagan, and D. G. Stone Br Dent J 2000; 188: 680-686
}

\begin{abstract}
Objective
The aim of these experiments was to compare the cutting dynamics of high-speed high-torque (speed-increasing) and high-speed lowtorque (air-turbine) handpieces and evaluate the effect of handpiece torque and bur type on sub-surface enamel cracking. Temperature changes were also recorded in teeth during cavity preparation with high and low torque handpieces with diamond and tungsten carbide (TC) burs. The null hypothesis of this study was that high torque handpieces cause more damage to tooth structure during cutting and lead to a rise in temperature within the pulp-chamber.
\end{abstract}

\section{Materials and methods \\ Images of the dynamic interactions between burs and enamel were recorded at video rate using a confocal microscope. Central incisors were mounted on a specially made servomotor driven stage for cutting with a type $57 \mathrm{TC}$ bur. The two handpiece types were used with simultaneous recording of cutting load and rate. Sub-surface enamel cracking caused by the use of diamond and TC burs with high and low torque was also examined. Lower third molars were sectioned horizontally to remove the cusp tips and then the two remaining crowns cemented together with cyanoacrylate adhesive, by their flat surfaces. Axial surfaces of the crowns were then prepared with the burs and handpieces. The teeth were then separated and the original sectioned surface examined for any cracks using a confocal microscope. Heat generation was measured using thermocouples placed into the pulp chambers of extracted premolars, with diamond and TC burs /high-low torque handpiece variables, when cutting occlusal and cervical cavities.}

\section{Results}

When lightly loaded the two handpiece types performed similarly. However, marked differences in cutting mechanisms were noted when increased forces were applied to the handpieces with, generally, an increase in cutting rate. The air turbine could not cope with steady heavy loads, tending to stall. 'Rippling' was seen in the interface as this stall developed, coinciding with the bur 'clearing' itself. No differences were noted between different handpieces and burs, in terms of sub-surface enamel cracking. Similarly, no differences were recorded for temperature rise during cavity preparation.

\section{Conclusions}

Differences in cutting mechanisms were seen between handpieces with high and low torque, especially when the loads and cutting rates were increased. The speed increasing handpiece was better able to cope with increased loading. Nevertheless, there was no evidence of increased tooth cracking or heating with this type of handpiece, indicating that these do not have any deleterious effects on the tooth.

\section{In brief}

- There are significant differences in cutting mechanism between air-turbines and speed-increasing handpieces that are particularly notable when a turbine is close to stalling under heavy cutting loads. The high-torque, speed-increasing handpiece is capable of much steadier cutting, giving better tactile feedback over the whole speed/loading range.

- Despite these differences in the mechanism of cutting there are no differences in sub-surface cracking of the tooth or in heat generation by the two types of handpieces.

- Modern handpieces with efficient water sprays cause an overall cooling of the teeth when cutting. Diamond burs caused slightly greater heat generation than tungsten carbide burs.

\section{Comment}

The development of the air-turbine 1 changed forever the way in which dental hard tissues were removed and this instrument has become the mainstay of operative dental procedures. There are disadvantages to the use of these high-speed low-torque machines, however, not least the lack of tactile feedback. The use of a hightorque handpiece run from a high-speed electric motor may increase tactile awarenesss but may cut enamel in a different way thereby causing more damage to the tooth.

This paper describes the use of sophisticated technology to record in real time the effects of cutting of tooth hard tissue with both a low-torque and high-torque handpiece. Confocal microscopic examination allowed analysis of the sub-surface enamel to investigate the presence of potentially damaging cracks. Finally, comparisons were made of the rise in temperature with- in the tooth between the two handpieces. The speed of advance of the bur was varied from $1 \mathrm{~mm}$ per minute to $5 \mathrm{~mm}$ per minute.

Cutting using the low-speed high-torque handpiece was comparable with the highspeed low-torque handpiece when the bur was advanced slowly. Increasing the rate of advance of the bur in the air-turbine handpiece tended to cause stalling. There were no such difficulties for the high torque handpiece. There was no difference in subsurface cracking between the two handpiece types. When the temperature within the pulp chamber was measured there was no significant difference between the two handpieces. The use of water coolant significantly reduced the temperature and the diamond bur generated more heat than the tungsten carbide bur.

The stalling that occurs with the air-turbine when subjected to higher loads is well known and hence the recommendation to use this handpiece with light brushstrokes. The use of the high torque handpiece would allow greater loads to be applied while not necessarily inducing iatrogenic damage to the dental pulp or hard tissues. Despite using considerable forces with the high torque speed-increasing handpiece the temperature within the pulp chamber did not increase above $35^{\circ} \mathrm{C}$. The use of suitable water coolant at the cutting site of the bur is known to reduce the likelihood of irreversible changes in the dental pulp. Interestingly, the tungsten carbide bur produced less heat than the diamond bur and this was attributed to the increased area of contact to tooth with the latter.

Professor W P Saunders

Professor of Endodontology,

University of Dundee 